\title{
A new approach to perturbation theory for a Dirac particle in a central field*
}

\author{
I.V. Dobrovolska and R.S. Tutik ${ }^{\dagger}$
}

April 19, 2022

PACS numbers: 03.65G, 03.65S.

Department of Physics, Dniepropetrovsk State University, Dniepropetrovsk, UA-320625, Ukraine.

ABstract. The explicit semiclassical treatment of logarithmic perturbation theory for the bound-state problem within the framework of the Dirac equation is developed. Avoiding disadvantages of the standard approach in the description of exited states, new handy recursion formulae with the same simple form both for ground and exited states have been obtained. As an example, the perturbation expansions for the energy eigenvalues for the Yukawa potential containing the vector part as well as the scalar component are considered.

\footnotetext{
${ }^{*}$ To be published in Physics Letters A 260 (1999) 10-16

${ }^{\dagger}$ Author to whom all correspondence should be addressed. E-mail address: tutik@ff.dsu.dp.ua
} 
Spectrum analysis poses some of the most important problems in quantum mechanics. Success of nonrelativistic potential models of quark confinement [1] reattracted attention to the bound-state problem in relativistic physics as well $[2,3]$. Several attempts have been made to describe relativistic systems in a central field due to a Lorentz-vector and Lorentz-scalar interaction within the framework of the Dirac equation. However, for almost all potentials this equation is not exactly solvable which compels to resort to some approximation methods.

A number of such approaches to solving the radial Dirac equation in analytical expressions have been developed, including, in particular, the use of the WKB-method [4,5], the hypervirial and Hellman-Feynman theorem [6,7], the $1 / N$-expansion [8-17], the algebraic approach [18], the method of Regge trajectories [19-21] and various perturbation schemes [21-26].

Despite such a variety of methods one of the most popular techniques is still remained logarithmic perturbation theory [27-32]. This technique involves reducing the Dirac equation, which becomes a pair of coupled first-order differential equations in central-field problems, to a nonlinear Riccati equation. In the case of ground states, the consequent expansion in a small parameter results in handy recursion relations which permit us to derive the high order corrections to energy eigenvalues and eigenfunctions. It should be emphasized that high orders of expansions are needed for applying modern summation procedures because the obtained perturbation series are typically divergent. However, when radially exited states are considered, the standard approach becomes extremely cumbersome and, practically, inapplicable. It is caused by factoring out zeros of the wave functions, which, in addition, are not the same zeros for small- and large-components of the Dirac spinor [33].

On the other hand, it is known, that the radial quantum number, $n_{r}$, most conveniently and naturally is introduced in consideration by means of quantization conditions, as in the WKB-approach [34,35]. However, since the WKB-approximation is more suitable for obtaining energy eigenvalues in the limiting case of large quantum numbers and the perturbation theory, on the contrary, deals with low-lying levels, the WKB quantization conditions need change.

Recently, a new technique based on a specific quantization condition has been proposed to get the perturbation series via semiclassical expansions within the one-dimensional Schrödinger equation [36]. For the Dirac equation, on performing the scale transformation, $r \rightarrow \hbar^{2} r$, the coupling constants appear in common with powers of Planck's constant, $\hbar$, thus implying the possibility to obtain perturbation expansions in a semiclassical manner in this case, too.

The objective of this letter is to develop the explicit semiclassical treatment of logarithmic perturbation theory for the bound-state problem within the framework of the Dirac equation and to describe a new procedure for deriving perturbation corrections through handy recursion formulae having the same simple form both for ground and exited states.

The proposed technique can be regarded as a further investigation of a part assigned to a rule of achieving a classical limit in construction of semiclassical methods. In addition to the rule, $\hbar \rightarrow 0, n_{r} \rightarrow \infty, l \rightarrow \infty$, $\hbar n_{r}=$ const, $\hbar l=$ const, required within the WKB-approach; and conditions, $\hbar \rightarrow 0, n_{r}=$ const, $l \rightarrow \infty$, $\hbar n_{r} \rightarrow 0, \hbar l=$ const, which are applied within the method of $1 / \mathrm{N}$-expansion [17]; here we address ourselves to the alternative possibility: $\hbar \rightarrow 0, n_{r}=$ const, $l=$ const, $\hbar n_{r} \rightarrow 0, \hbar l \rightarrow 0$, that results in the explicit semiclassical treatment of the logarithmic perturbation theory.

(1) Method. We study the bound state problem for a single fermion moving in an attractive central potential. This potential contains both the time component of a Lorentz four-vector, $V(r)$, and a Lorentz-scalar term, $W(r)$, which, in general, have a Coulomb-like behaviour at 
the origin

$$
V(r)=\frac{1}{r} \sum_{i=0}^{\infty} V_{i} r^{i}, W(r)=\frac{1}{r} \sum_{i=0}^{\infty} W_{i} r^{i}
$$

though the case $V_{0}=0$ or $W_{0}=0$ is permissible, too. In what follows, a scalar potential will be included in the mass term $m(r)$ by analogy with "dynamical mass" models of quark confinement $[2,3]$

$$
m(r)=m+\frac{W(r)}{c^{2}}
$$

Then the Dirac radial wave equations have the form

$$
\begin{aligned}
& \hbar F^{\prime}(r)-\frac{\hbar \chi}{r} F(r)+\frac{1}{c}\left[E-V(r)-m(r) c^{2}\right] G(r)=0, \\
& \hbar G^{\prime}(r)+\frac{\hbar \chi}{r} G(r)-\frac{1}{c}\left[E-V(r)+m(r) c^{2}\right] F(r)=0 .
\end{aligned}
$$

Here $F(r)$ and $G(r)$ are the small and large components of the wavefunction of a particle and $E$ is its total energy, $\chi=s\left(j+\frac{1}{2}\right)$ for $j=l-\frac{s}{2}$, with $s= \pm 1$ denoted the sign of $\chi$.

Eliminating $F(r)$ from the system (3) and performing the substitution, $R(r)=\hbar G^{\prime}(r) / G(r)$, for the logarithmic derivative of large component we then arrive at the Riccati equation

$$
\begin{aligned}
& \hbar R^{\prime}(r)-\hbar Q(r) R(r)+R^{2}(r) \\
& =\frac{\hbar^{2} \chi(\chi+1)}{r^{2}}+Q(r) \frac{\hbar^{2} \chi}{r}+m^{2}(r) c^{2}-\frac{1}{c^{2}}[E-V(r)]^{2},
\end{aligned}
$$

with $Q(r)=\left[m^{\prime}(r)-V^{\prime}(r)\right] /[E+m(r)-V(r)]$.

As was above pointed out, logarithmic perturbation theory does involve coupling constants in common with powers of Planck's constant. Therefore we attempt now to solve eq.(4) in a semiclassical manner. Taking into account the leading orders in $\hbar$ of the quantities $E \sim \frac{1}{\hbar^{2}}$, $\hbar \chi \sim \hbar$, from Riccati equation (4) we have

$$
\begin{aligned}
& R(r)=\frac{1}{\hbar} \sum_{i=0}^{\infty} R_{i}(r) \hbar^{2 i} \\
& Q(r)=\hbar^{2} \sum_{i=0}^{\infty} Q_{i}(r) \hbar^{2 i}, \\
& E=\frac{1}{\hbar^{2}} \sum_{i=0}^{\infty} E_{i} \hbar^{2 i} .
\end{aligned}
$$

Keeping in mind that relativistic mechanics must go over to nonrelativistic one as the speed of light tends to infinity and quantum mechanics must go over to classical one as $\hbar \rightarrow 0$, the correlation between these constants has to be set. By analogy with quantum electrodynamics the foregoing consideration will be carried out under the condition $\hbar c \sim O(1)$ [37]. Moreover, for simplicity we put $\hbar c=1$.

On substituting the expansions (5) into the Riccati equation (4) and comparing coefficients 
of the different powers of $\hbar$, one obtains the following hierarchy of equations

$$
\begin{aligned}
& R_{0}^{2}(r)=m^{2}-E_{0}^{2}, \\
& R_{0}(r) R_{1}(r)=E_{0}\left[V(r)-E_{1}\right]+m W(r), \\
& R_{1}^{\prime}(r)+2 R_{0}(r) R_{2}(r)+R_{1}^{2}(r)-R_{0}(r) Q_{0}(r) \\
& =\frac{\chi(\chi+1)}{r^{2}}+2 E_{1} V(r)-E_{1}^{2}-2 E_{0} E_{2}+W^{2}(r)-V^{2}(r), \\
& \ldots \\
& R_{k-1}^{\prime}(r)+\sum_{j=0}^{k} R_{j}(r) R_{k-j}(r)-\sum_{j=0}^{k-2} R_{j}(r) Q_{k-j-2}(r) \\
& =2 E_{k-1} V(r)-\sum_{j=0}^{k} E_{j} E_{k-j}+\frac{\chi}{r} Q_{k-3}(r),
\end{aligned}
$$

where

$$
\begin{aligned}
& Q_{0}(r)=\frac{1}{E_{0}+m}\left[W^{\prime}(r)-V^{\prime}(r)\right], \\
& Q_{k}(r)=-\frac{1}{E_{0}+m}\left[\sum_{j=0}^{k-1} Q_{j}(r) E_{k-j}+Q_{k-1}(r)[W(r)-V(r)]\right] .
\end{aligned}
$$

For nodeless states this system can be solved straightforwardly. However, when radial excitations are described with standard technique the nodes of the wave functions need to be factored out first and consideration becomes extremely cumbersome. We intend to circumvent this difficulty by making use of the quantization condition. Its fundamental idea that stems from the WKB-approach $[34,35]$ is well known as the principle of argument in the analysis of complex variables. Being applied to the logarithmic derivative, $R(r)$, it means that

$$
\frac{1}{2 \pi \mathrm{i}} \oint R(r) \mathrm{d} r=\hbar N
$$

where $N$ is a number of zeros inside a closed contour.

This condition is exact and is widely used for deriving the high-order corrections to the WKB-approximation and the $1 / N$-expansions. There is, however, one important point to note. The radial and orbital quantum numbers, $n_{r}$ and $l$, correspondingly, are specific quantum notions and need be defined before going over from quantum mechanics to classical physics. Therefore the quantization condition (7) must be supplemented with the rule of achieving a classical limit that stipulates the type of semiclassical approximation.

In particular, within the WKB-approach the passage to the classical limit is implemented using the rule

$$
\hbar \rightarrow 0, n_{r} \rightarrow \infty, l \rightarrow \infty, \hbar n_{r}=\text { const, } \hbar l=\mathrm{const},
$$

whereas the $1 / N$-expansion, being complementary to the WKB-method, requires the conditions $[17]$

$$
\hbar \rightarrow 0, n_{r}=\text { const, } l \rightarrow \infty, \hbar n_{r} \rightarrow 0, \hbar l=\text { const. }
$$

The semiclassical treatment of logarithmic perturbation theory proved to ivolve the alternative possibility:

$$
\hbar \rightarrow 0, n_{r}=\text { const, } l=\text { const, } \hbar n_{r} \rightarrow 0, \hbar l \rightarrow 0 .
$$

Notice that the part of this rule concerned the orbital quantum number, $l$, differs from one used within the WKB-approach and the $1 / N$-expansion method. In our consideration this part, implying the first order in $\hbar$ for the quantity $\hbar \chi$, has been used in deriving the system (6). 
The remaining part of the rule respects the radial quantum number. Due to (10) the righthand side of the equality (7) has the first order in $\hbar$ and, hence, on substituting the expansion (5) the quantization conditions (7) takes the form

$$
\frac{1}{2 \pi \mathrm{i}} \oint R_{i}(r) \mathrm{d} r=N \delta_{i, 1},
$$

where the Kronecker delta $\delta_{i j}$ is used.

Before proceeding further, we must specify the quantity $N$. In contrast to the WKBapproach and the $1 / N$-method, we choose such a contour of integration which encloses both the nodes of the wave function $G(r)$ and the boundary point, $r=0$. Then the quantity $\mathrm{N}$ is depends on both the radial quantum number, $n_{r}$, and the behaviour of the wave function near the origin, and is given by

$$
N=n_{r}+\sqrt{\chi^{2}+W_{0}^{2}-V_{0}^{2}}, n_{r}=n+(s+1) / 2, n=0,1,2, \ldots
$$

A further application of the theorem of residues to the explicit form of functions $R_{i}(r)$ easily solves the problem of taking into account nodes of the wave functions for exited states.

(2) Recursion formulae. We begin with investigation of behaviour of the functions $R_{i}(r)$. From the system (6) we have

$$
R_{0}(r)=-\sqrt{m^{2}-E_{0}^{2}},
$$

where the minus sign is chosen from boundary condition. Then the function $R_{1}(r)$ has a simple pole at the origin, owing to the Coulombic behaviour of the potentials at this point, while the function $R_{k}(r)$ has a pole of the order $k$. Hence $R_{k}(r)$ can be represented by the Laurent series

$$
R_{k}(r)=r^{-k} \sum_{i=0}^{\infty} R_{i}^{k} r^{i}
$$

which makes it possible to write the quantization conditions (11) as

$$
R_{k}^{k+1}=N \delta_{k, 0} .
$$

Now, by analogy with $R_{k}(r)$, let us also represent the functions $Q_{k}(r)$, involved in the expansion (5), as a power series in $r$ :

$$
Q_{k}(r)=r^{-2-k} \sum_{i=0}^{\infty} Q_{i}^{k} r^{i} .
$$

From the equation $Q(r)[E+m(r)-V(r)]=m^{\prime}(r)-V^{\prime}(r)$ we then obtain

$$
\begin{aligned}
Q_{i}^{0} & =\frac{i-1}{E_{0}+m}\left(W_{i}^{0}-V_{i}^{0}\right), \\
Q_{i}^{k} & =-\frac{1}{E_{0}+m}\left[\sum_{j=0}^{k-1} Q_{j+i-k}^{j} E_{k-j}+\sum_{j=0}^{i} Q_{j}^{k-1}\left(W_{i-j}-V_{i-j}\right)\right] .
\end{aligned}
$$

Finally, substituting expansions (14) and (16) into the last equation of the system (6) and collecting coefficients of the like powers of $r$ leads to the recursion relation in terms of the 
Laurent coefficients, $R_{i}^{k}$ :

$$
\begin{aligned}
& R_{i}^{k}=-\frac{1}{2 R_{0}^{0}}\left[(i-k+1) R_{i}^{k-1}+\sum_{j=1}^{k-1} \sum_{p=0}^{i} R_{p}^{j} R_{i-p}^{k-j}-\sum_{j=0}^{k-2} \sum_{p=0}^{i} Q_{p}^{j} R_{i-p}^{k-2-j}\right. \\
& -\chi Q_{i}^{k-3}+\delta_{k, i} \sum_{j=0}^{k} E_{j} E_{k-j}-2 E_{k-1} V_{i-k+1} \\
& \left.+\delta_{k, 2} \sum_{p=0}^{i}\left(V_{p} V_{i-p}-W_{p} W_{i-p}\right)-\delta_{k, 1} 2 m W_{i}-\delta_{i, 0} \delta_{k, 2} \chi(\chi+1)\right],
\end{aligned}
$$

where for universality of designations we put $R_{0}^{0}=R_{0}, R_{i}^{0}=0, i>0$.

In the case $i \neq k$, this formula is intended for obtaining coefficients $R_{i}^{k}$. When $i=k$, by equating the explicit expression for $R_{k}^{k+1}$ to the quantization condition (15) we get the recursion relation for the energy eigenvalues

$$
\begin{aligned}
& E_{0}=\frac{m}{N^{2}+V_{0}^{2}}\left(N \sqrt{N^{2}+V_{0}^{2}-W_{0}^{2}}-V_{0} W_{0}\right), k=0, \\
& E_{k}=\frac{R_{0}^{0} R_{0}^{1}}{2\left(E_{0} R_{0}^{1}+V_{0} R_{0}^{0}\right)}\left[\frac { 1 } { R _ { 0 } ^ { 1 } } \left(\sum_{j=2}^{k-1} \sum_{p=0}^{k} R_{p}^{j} R_{k-p}^{k+1-j}+2 \Theta(k-2) \sum_{p=1}^{k} R_{p}^{1} R_{k-p}^{k}\right.\right. \\
& \left.-\sum_{j=0}^{k-1} \sum_{p=0}^{k} Q_{p}^{j} R_{k-p}^{k-1-j}+\delta_{k, 1}\left(V_{0} V_{1}-W_{0} W_{1}\right)-\chi Q_{k}^{k-2}\right) \\
& -\frac{1}{R_{0}^{0}}\left(R_{k}^{k-1}+\sum_{j=1}^{k-1} \sum_{p=0}^{k} R_{p}^{j} R_{k-p}^{k-j}-\sum_{j=0}^{k-2} \sum_{p=0}^{k} Q_{p}^{j} R_{k-p}^{k-2-j}\right. \\
& +\sum_{j=1}^{k-1} E_{j} E_{k-j}-2 E_{k-1} V_{1}+\delta_{k, 2} \sum_{p=0}^{2}\left(V_{p} V_{2-p}-W_{p} W_{2-p}\right) \\
& \left.\left.-\delta_{k, 1} 2 m W_{1}-\chi Q_{k}^{k-3}\right)\right], k>0 .
\end{aligned}
$$

Here $E_{0}$ does be the exact solution to the Dirac-Coulomb equation $[38,39]$ and we use the step function

$$
\begin{aligned}
\Theta(k) & =1, \quad k \geq 0, \\
& =0, \quad k<0 .
\end{aligned}
$$

Thus, equations (18) and (19) determine the coefficients of perturbation expansions of energy eigenvalues and eigenfunctions for screened Coulomb potentials in the same form both for ground and exited states.

(3) Examples of application. As a check of the obtained formulae we calculate the energy eigenvalues for the pure-vector, screened Coulomb potential of general form. On applying the recursion relations (18) and (19), analytical expressions for the perturbation coefficients are 
found to be equal to

$$
\begin{aligned}
E_{0}= & \frac{m N}{\sqrt{N^{2}+a^{2}}} \\
E_{1}= & a V_{1} \\
E_{2}= & -\frac{V_{2}}{2 \rho^{2}}\left(3 a^{2} \epsilon-\chi(\chi \epsilon+1) \rho^{2}\right) \\
E_{3}= & \frac{V_{3}}{2 \rho^{4}}\left(a^{3}\left(4 \epsilon^{2}+1\right)-a\left(2 \chi^{2} \epsilon^{2}+3 \chi \epsilon+\chi^{2}-1\right) \rho^{2}\right) \\
E_{4}= & \frac{1}{8 \rho^{6}}\left(V _ { 2 } ^ { 2 } \left[a^{4} \epsilon\left(5 \epsilon^{2}-12\right)+a^{2} \epsilon\left(6 \chi^{2} \rho^{2}-5\right) \rho^{2}\right.\right. \\
& \left.+\chi^{2}\left[\chi^{2} \epsilon\left(\epsilon^{2}+2\right)+\chi\left(4 \epsilon^{2}+2\right)+3 \epsilon\right] \rho^{4}\right] \\
& +V_{4}\left[-5 a^{4} \epsilon\left(4 \epsilon^{2}+3\right)+a^{2}\left[6 \chi^{2} \epsilon\left(2 \epsilon^{2}+3\right)+6 \chi\left(4 \epsilon^{2}+1\right)-25 \epsilon\right] \rho^{2}\right. \\
& \left.\left.-3 \chi\left(\chi^{2}-1\right)(\chi \epsilon+2) \rho^{4}\right]\right) \\
E_{5}= & \frac{-a}{8 \rho^{8}}\left(V _ { 2 } V _ { 3 } \left[3 a^{4}\left(8 \epsilon^{4}-20 \epsilon^{2}-3\right)-a^{2}\left[\chi^{2}\left(32 \epsilon^{4}-36 \epsilon^{2}-10\right)\right.\right.\right. \\
& \left.+\chi \epsilon\left(10 \epsilon^{2}-24\right)+9\left(6 \epsilon^{2}+1\right)\right] \rho^{2}+\chi\left[30 \chi^{2} \epsilon^{3}\right. \\
& \left.\left.+24 \chi \epsilon^{2}+10 \epsilon+\chi+\chi^{3}\left(8 \epsilon^{4}+8 \epsilon^{2}-1\right)\right] \rho^{4}\right] \\
& +V_{5}\left[-3 a^{4}\left(8 \epsilon^{4}+12 \epsilon^{2}+1\right)+a^{2}\left[\chi^{2}\left(16 \epsilon^{4}+48 \epsilon^{2}+6\right)\right.\right. \\
& \left.+10 \chi \epsilon\left(4 \epsilon^{2}+3\right)-15\left(6 \epsilon^{2}+1\right)\right] \rho^{2} \\
& \left.\left.+\left[5 \chi^{2}\left(4 \epsilon^{2}+3\right)-3 \chi^{4}\left(4 \epsilon^{2}+1\right)+50 \chi \epsilon-30 \chi^{3} \epsilon-12\right] \rho^{4}\right]\right),
\end{aligned}
$$

where $a=V_{0}, \epsilon=E_{0}$ and $\rho$ is $\sqrt{1-\epsilon^{2}}$.

One can verifies that the first three corrections coincide with those derived by McEnnan et al [24] with standard technique.

The next example will be the attractive Yukawa potential, often utilized in relativistic calculations, which has not only the Lorentz-vector component, $V(r)=-(a / r) e^{-\lambda r}$, but the Lorentz-scalar term, $W(r)=-(b / r) e^{-\mu r}$, as well. Now the analytic expressions for perturba- 
tion corrections to the bound state energy take the form

$$
\begin{aligned}
E_{0}= & \frac{m}{N^{2}+a^{2}}\left(N \sqrt{N^{2}+a^{2}-b^{2}}-a b\right) \\
E_{1}= & a \lambda+b \mu \epsilon \\
E_{2}= & -\frac{1}{4(b \epsilon+a) \rho^{2}}\left(\lambda ^ { 2 } \left[3 a^{3} \epsilon-a \chi(\chi \epsilon+1) \rho^{2}+2 a^{2} b\left(2 \epsilon^{2}+1\right)\right.\right. \\
& \left.+a b^{2} \epsilon\left(\epsilon^{2}+2\right)\right] \\
& \left.+\mu^{2}\left[3 b^{3} \epsilon^{2}-b \chi \epsilon \rho^{2}-b \chi^{2} \rho^{2}+2 b^{2} a \epsilon\left(\epsilon^{2}+2\right)+b a^{2}\left(2 \epsilon^{2}+1\right)\right]\right), \\
E_{3}= & \frac{1}{12(a+b \epsilon) \rho^{4}}\left(\lambda ^ { 3 } \left[a^{4}\left(4 \epsilon^{2}+1\right)-a^{2}\left(2 \chi^{2} \epsilon^{2}+3 \chi \epsilon+\chi^{2}-1\right) \rho^{2}\right.\right. \\
& +3 a^{3} b \epsilon\left(2 \epsilon^{2}+3\right)+a^{2} b^{2}\left(2 \epsilon^{4}+11 \epsilon^{2}+2\right)-a b\left(3 \chi+\left(3 \chi^{2}-1\right) \epsilon\right) \rho^{2} \\
& \left.+a b^{3} \epsilon\left(3 \epsilon^{2}+2\right)\right] \\
& +\mu^{3}\left[b^{4}\left(-8 \epsilon^{4}+13 \epsilon^{2}\right)+b^{2}\left(3 \chi \epsilon^{3}+\left(2 \chi^{2}+1\right) \epsilon^{2}-6 \chi \epsilon-5 \chi^{2}\right) \rho^{2}\right. \\
& -3 b^{3} a \epsilon\left(2 \epsilon^{4}-\epsilon^{2}-6\right)-b^{2} a^{2}\left(4 \epsilon^{4}-14 \epsilon^{2}-5\right) \\
& \left.-b a \epsilon\left(3 \chi \epsilon+3 \chi^{2}-1\right) \rho^{2}+b a^{3} \epsilon\left(2 \epsilon^{2}+3\right)\right] \\
& +\lambda^{2} \mu\left[9 a^{3} b \epsilon \rho^{2}-6 a^{2} b^{2}\left(2 \epsilon^{4}-\epsilon^{2}-1\right)\right. \\
& \left.\left.+3 a b^{3} \epsilon \rho^{2}\left(\epsilon^{2}+2\right)-3 a b \chi(\chi \epsilon+1) \rho^{4}\right]\right) .
\end{aligned}
$$

In order to assess the speed and accuracy of the perturbation technique for the Yukawa potential with various ratios of its component we consider energy eigenvalues for the pure vector case, $V(r)=-(a / r) e^{-\lambda r}$; the pure scalar potential, $W(r)=-(a / r) e^{-\lambda r}$; and the equally mixed interaction, $V(r)+W(r)=-\left(I+\gamma_{0}\right)(a / 2 r) e^{-\lambda r}$. Typical results of calculation are represented in Table 1 where the sequence of the sums of first terms from our expansion for relativistic binding energies is compared with the results, $E_{\text {num }}$ (in $\mathrm{KeV}$ ), obtained by numerical integration. The calculation has been performed for $s=1, n_{r}=1, l=1$ and $s=-1, n_{r}=1, l=0$ states with parameters $a=\alpha z, \lambda=1,13 \alpha z^{1 / 3}, z=74$ ( $\alpha$ is the fine-structure constant and $z$ the nuclear charge).

As it can be seen from Table 1, in all cases we have two subsequences bounded below and above the energy eigenvalues. The average of these subsequences at the point of their maximal drawing together is proved to result in a quite good approximation to the exact value.

To summarize, we have developed a semiclassical treatment of logarithmic perturbation theory for a Dirac particle in a central field. Based upon the $\hbar$-expansions and suitable quantization conditions, new handy recursion relations for solving the bound-state problem for the Dirac equation with the screened Coulomb potential having both vector and scalar component have been derived. Avoiding the disadvantages of the standard approach these formulae have the same simple form both for ground and exited states and provide, in principle, the calculation of the perturbation corrections up to an arbitrary order in the analytic or numerical form. And at last, this approach does not imply knowledge of the exact solution for zero approximation, which is obtained automatically. 
This work was supported in part by the International Soros Science Education Program (ISSEP) under grant APU052102. 


\section{References}

[1] C.Quigg and J.L. Rosner, Rhys. Rep. 56 (1979) 167.

[2] C.L. Critchfield, Phys. Rev. D12 (1975) 923.

[3] J.F. Gunion and L.F. Li, Phys. Rev. D12 (1975) 3583.

[4] R.H. Good, Jr., Phys. Rev. 90 (1953) 131; 94(154) 931.

[5] Kosaka Keiji and Yonei Katsumi, J. Phys. Soc. Japan 60 (1991) 850.

[6] M. Brack, Phys.Rev. D27 (1983) 1950.

[7] E.R. Vrscay and H. Hamidian, Phys. Lett. A130 (1988) 141.

[8] R. Roychoudhuri and Y.P. Varshni, J. Phys. A20 (1987) L1083.

[9] M.M. Panja and R. Dutt, Phys.Rev. A38 (1988) 3937.

[10] A. Atag, J. Math. Phys. 30 (1989) 696.

[11] R. Roychoudhuri and Y.P. Varshni, Phys.Rev. A39 (1989) 5523.

[12] A. Chatterjee, Rhys. Rep.186 (1990) 249.

[13] M.M. Panja, R. Dutt and Y.P. Varshni, Phys.Rev. A42 (1990) 106.

[14] B. Roy and R. Roychoudhuri, J. Phys. A23 (1990) 3555.

[15] D.Z. Goodson, J.D. Morgan and D.R. Herschbach, Phys.Rev. A43 (1991) 4617.

[16] E. Papp, Ann. Phys. (Leipzig) 48 (1991) 319.

[17] S.S. Stepanov and R.S. Tutik, Phys. Lett. A163 (1992) 26.

[18] B. Roy and R. Roychoudhuri, J. Phys. A23 (1990) 5095.

[19] H.J.W. Müller, Z. Phys. 183 (1965) 402.

[20] S.S. Stepanov and R.S. Tutik, Preprint ITP-92-14E (Kiev, 1992).

[21] L.K. Sharma, S. Mechta and V.P. Iyer, J. Math. Phys. 24 (1983) 2521.

[22] G. Breit and G.E. Broun, Phys. Rev. 76 (1949) 1307.

[23] R.A. Moore, Can. J. Phys. 53 (1975) 1240.

[24] J. McEnnan, D.J. Botto and R. H. Pratt, Phys.Rev. A16 (1977) 1768.

[25] S.G. Davidson, R.J. Jerrard, C.L. Roy and M.L. Glasser, Indian J. Pure \& Appl. Phys. 22 (1984) 65.

[26] E. Papp, Phys.Rev. A33 (1986) 719. 
[27] A.I. Mikhailov and V.S. Polikanov Zh. Eksp. Teor. Fiz. 54 (1968) 175 [Sov. Phys.-JETP 27 (1968) 95].

[28] C.K. Au and G.W. Rogers, Phys.Rev. A22 (1980) 1820.

[29] C.K. Au, Phys.Rev. A23 (1981) 2068.

[30] G.W. Rogers, Phys.Rev. A30 (1984) 35.

[31] A.V. Sergeev and A.I. Sherstyuk, Yad. Fiz. 39 (1984) 1158 [Sov. J. Nucl. Phys. 39 (1984) $731]$.

[32] G.W. Rogers, J. Math. Phys. 26 (1985) 567.

[33] I.P. Grant, Adv. Phys. 19 (1979) 747.

[34] A. Zwaan, Arch. Neerland. Sci. Exact. Natur. Ser. 3A12 (1929) 1.

[35] J.L. Dunham, Phys.Rev. 41(1932) 713.

[36] I.V. Dobrovolska and R.S. Tutik, J. Phys. A32 (1999) 563.

[37] I. Bialynicki-Birula and Z. Bialynicka-Birula, Quantum Electrodynamics (PWN, Warszawa, 1969) (In Polish).

[38] W. Greiner, B. Müller and J. Rafelski, Quantum Electrodynamics of Strong Fields (Springer, New York, 1985).

[39] R.S. Tutik, J. Phys. A25 (1992) L413. 
Table 1

\begin{tabular}{c|lll|lll}
\hline \multirow{2}{*}{$\mathrm{k}$} & \multicolumn{3}{|c|}{$s=1, n_{r}=1, l=1$} & \multicolumn{3}{c}{$s=-1, n_{r}=1, l=0$} \\
\cline { 2 - 7 } & $E_{V}$ & $E_{W}$ & $E_{V+W}$ & $E_{V}$ & $E_{W}$ & $E_{V+W}$ \\
\hline 0 & 20.644616 & 16.59173 & 18.292804 & 20.644616 & 16.59173 & 18.292804 \\
1 & 11.091653 & 7.348948 & 10.846326 & 11.091653 & 7.348948 & 10.846326 \\
2 & 12.415123 & 9.066156 & 11.810855 & 12.721342 & 9.372375 & 12.003761 \\
3 & 12.264120 & 8.784152 & 11.703753 & 12.500951 & 8.995036 & 11.855249 \\
4 & 12.308677 & 8.880691 & 11.730813 & 12.558797 & 9.118034 & 11.890052 \\
5 & 12.292914 & 8.838096 & 11.722213 & 12.537837 & 9.063240 & 11.878837 \\
6 & 12.299805 & 8.860978 & 11.725555 & 12.546910 & 9.092488 & 11.883163 \\
7 & 12.296473 & 8.847251 & 11.724110 & 12.542466 & 9.074820 & 11.881275 \\
8 & 12.298236 & 8.856234 & 11.724792 & 12.544844 & 9.086483 & 11.882175 \\
9 & 12.297242 & 8.849965 & 11.724449 & 12.543482 & 9.078246 & 11.881715 \\
10 & 12.297834 & 8.854576 & 11.724631 & 12.544306 & 9.084385 & 11.881963 \\
11 & 12.297465 & 8.851033 & 11.724530 & 12.543784 & 9.079601 & 11.881823 \\
12 & 12.297704 & 8.853860 & 11.724588 & 12.544128 & 9.083474 & 11.881905 \\
13 & 12.297544 & 8.851529 & 11.724553 & 12.543893 & 9.080233 & 11.881856 \\
14 & 12.297654 & 8.853509 & 11.724575 & 12.544058 & 9.083025 & 11.881886 \\
15 & 12.297576 & 8.851782 & 11.724561 & 12.543939 & 9.080555 & 11.881867 \\
\hline$E_{\text {num }}$ & 12.297609 & 8.852592 & 11.724567 & 12.543990 & 9.081723 & 11.881875 \\
\hline
\end{tabular}

